\title{
Pair Analytics: Capturing Reasoning Processes in Collaborative Visual Analytics
}

\author{
Arias-Hernandez, Richard \\ Simon Fraser University \\ ariasher@sfu.ca
}

\author{
Kaastra, Linda T. \\ University of British \\ Columbia - MAGIC \\ 1kaastra@magic.ubc.ca
}

\author{
Green, Tera M. \\ Simon Fraser University \\ terag@sfu.ca
}

\author{
Fisher, Brian \\ Simon Fraser University \\ bfisher@sfu.ca
}

\begin{abstract}
Studying how humans interact with abstract, visual representations of massive amounts of data provides knowledge about how cognition works in visual analytics. This knowledge provides guidelines for cognitive-aware design and evaluation of visual analytic tools. Different methods have been used to capture and conceptualize these processes including protocol analysis, experiments, cognitive task analysis, and field studies. In this article, we introduce Pair Analytics: a method for capturing reasoning processes in visual analytics. We claim that Pair Analytics offers two advantages with respect to other methods: (1) a more natural way of making explicit and capturing reasoning processes and (2) an approach to capture social and cognitive processes used to conduct collaborative analysis in real-life settings. We support and illustrate these claims with a pilot study of three phenomena in collaborative visual analytics: coordination of attention, cognitive workload, and navigation of analysis.
\end{abstract}

\section{Introduction}

The study of analytical reasoning and interaction with visual analytic tools is a critical step in the advancement of visual analytics as a scientific field [25]. Creating knowledge and models about higherorder cognitive processes in visual analytics [13] and the leverage points in which computational tools can amplify human cognition [24] is currently one of the most pressing challenges in laying out the scientific foundations for this endeavor. To be able to create this kind of knowledge researchers require the use of methods that allow them to capture and conceptualize the analyst's reasoning processes. Several methods have been used previously depending on the phenomenon of study and the ontological assumptions about the phenomenon. For example, studying "insight" in visual analytics can be approached by protocol analysis, by fMRI analysis, or by field studies depending on the conceptualization of "insight" as a verbalized observation about the data [28], a pattern of brain activation [3], or a social process [29]. Every research method used to capture reasoning processes comes with its own advantages and limitations. For example, take Protocol Analysis, a well established method in cognitive studies of scientific visualization [33], usability studies and evaluation in visual analytics $[30,19]$. Protocol Analysis provides access to data on thinking through verbal reports of participants about their thought processes [8]. However, it has been noted that once participants are immersed in the task at hand the amount of verbalization decreases and their reports on their thought process becomes scattered and fragmented [36]. This behavior compromises the completeness of the thinking data, making it necessary to use complementary methods (e.g. logging mechanisms) for purposes of cross-validity.

In this paper, we introduce "Pair Analytics," a research method for capturing reasoning processes in visual analytics that addresses some of the limitations of currently available methods. Pair analytics requires a dyad of participants: one Subject Matter Expert (SME) and one Visual Analytics Expert (VAE). These two participants are given one analytical task, one data set, and one computer with a visual analytics tool. Each participant is assigned a role. The VAE plays the role of the "driver" and the SME plays the role of the "navigator" of the VA tool. The pairing of VAE and SME in the context of an analytic task is designed to generate a human-human dialog that makes explicit the mental models being used and the analytic reasoning followed by each participant.

As a research protocol, pair analytics builds on the tradition of "in-vivo" studies of cognition $[33,15]$ and the strategy of pair programming from extreme programming software development methods [9]. We claim that pair analytics offers two advantages to other methods for capturing visual, analytical reasoning. 
First, pair analytics is a more natural way of making explicit and capturing reasoning processes. In pair analytics participants talk aloud to each other, without being prompted from a researcher or without previous training in the method. This kind of natural, human interaction allows speech to flow continuously avoiding the silence gaps that occur in protocol analysis. Pairing participants with different expertise and from different work environments also reduces the chances of tacit knowledge not being verbalized, one of the limitations of in-vivo studies of cognition in social settings [11]. Second, pair analytics makes evident certain cognitive processes used to structure and coordinate collaborative analysis. These kinds of processes cannot be captured by protocol analyses, or by any other individually-focused methods, since these are interactive processes and can only be captured in social, interactive settings. Theoretical frameworks from psychology and cognitive science, such as joint activity theory [6] can be used to observe and capture these social and psycholinguistic processes. We support these claims in this paper with a pilot study in the domain of aircraft safety and maintenance engineering that explores three cognitive phenomena: coordination of attention, cognitive workload, and structuring of analytical reasoning.

The first part of this paper describes pair analytics and situates the method with respect to other methods used to study analytical reasoning in visual analytics. The second part elaborates on the pilot study and the claims made about pair analytics. The last part presents the limitations of this method and future work that has to be conducted on pair analytics.

\section{Related work}

Several methodological approaches are used in the study of analytical reasoning in visual analytics. In this section we present the most commonly used methods highlighting some of their advantages and limitations.

\subsection{Protocol analysis}

Protocol Analysis, or the "think-aloud" method, elicits verbal reports from participants about their thought process when performing specific tasks [8]. This method is based on two assumptions. First, contents of working memory are uttered in speech, where they can be coded and analyzed [8]. Second, participants can be trained to make verbal reports of their thought process without altering the train of thought used in the completion of a task [8]. Protocol Analysis has been extensively used in visual analytics to study varied cognitive phenomena, among them: information foraging [23], thought experiments and spatial transformations [34,35], sensemaking [33], insight generation in bioinformatics [28], and analytical strategies used in intelligence analysis [19] and financial analysis [17]. This method requires a structured and validated coding scheme that characterizes the phenomenon to be captured from the verbal reports.

Protocol analysis offers several advantages, among them: a systematic way of tracing cognitive states and their dynamics, and detailed descriptions of goals, intentions and perceptions that drive behavior. A major caveat of this method is that once participants get absorbed in the task at hand, they tend to decrease their verbal reports [36]. Another reported limitation is that imposing think-aloud methods during analysis can affect reasoning processes, such as insight [31].

\subsection{Experiments}

Experiments designed to study the influence of cognitive factors on performance in visual analytics is another method previously used by researchers. In these experiments, the independent variables are usually the task, the data, the visualization or visual analytics tool, and type of participants. Dependent variables are usually accuracy and time of analysis. Results of these studies are quantitative and usually highlight whether the factor(s) studied influence performance in a statistically significant way or not. If the study incorporates correlation analyses, the results will consider the cognitive factors to be "predictive" or not of the phenomenon of interest (e.g. performance).

In visual analytics the use of experiments, or quasiexperiments (e.g. correlation studies), is more common in perceptual studies of vision [16], than in cognitive studies of analytical reasoning [25], in which they are very rare [12]. To account for the influence of cognitive factors, experiments sometimes make use of "inscription devices" [20] that track or monitor neurological, physiological or interactional states (e.g. fMRI, eye-tracking, logging mechanisms) and tests that characterize psychological profiles of individuals.

Some advantages of experiments are: scientific testing of hypotheses, statistical validity, generalized claims about a specific phenomenon, and reliability. Limitations of experiments include: limitations in the samples, which not necessarily use domain experts or center on non-representative populations; synthetic analytical tasks, which normally are close-ended, simple and unambiguous; synthetic data, which normally embodies a "ground truth;" and limitations in resembling real-life conditions for analytical work. Experiments, for example, have time constrains for visual analysis, normally a few hours, that do not 
reflect the duration of real-time analyses that can take several days or even months. The size of the datasets in experiments is usually bounded and it does not represent real-life situations in which size and scale of the data are not predetermined [27]. Other factors that structure analytical work in organizations and that normally do not make it to controlled experiments are: exploratory and open-ended analyses, interruptions, multiple information resources, interaction with peers, division of labor, integration of different information tools, and deep knowledge of the data, tools and tasks.

\subsection{Cognitive Task Analysis}

Cognitive Task Analysis (CTA) is a set of methods for identifying cognitive skills, or mental demands, needed to perform a task proficiently [21]. CTA requires prior knowledge of expert-related schemas required to perform a task. This knowledge is obtained through observation and interviews with experts at performing the task. Participants are then observed while working on the task to determine which of these schemas are more efficient and accurate. The schemas are then captured as tacit knowledge so that they can be used in training for effective performance. CTA is not a common method currently used in visual analytics. This is a consequence of the tendency of the field on focusing on understanding and improving the visual analytic tools rather than on understanding and improving the human side of the analytical equation. Some notable exceptions are Pirolli and Card's study on sensemaking [24] Wright et al.'s work on the sandbox for analysis [37], and Trafton's study on usage of qualitative mental models [33].

\subsection{Field studies}

Field, or "in-vivo," studies collect ethnographic data of cognition in action. These methods normally study cognition in realistic, non-controlled settings [7]. In the case of analytical reasoning, in-vivo studies require non-participant observation of domain experts in their workplace, working on genuine problems, and using their familiar data and tools. As a consequence of the radical critique made in the 1980 s by Lucy Suchman [32] to the information-processing paradigm in cognitive sciences, ethnomethods began drawing attention in cognitive studies [15,22]. However, in-vivo studies of analytic reasoning in visual analytic are still very rare. Some variants of in-vivo studies have been used in the study of insight as a long-term and social process in bioinformatics [29], confirmation bias as a social phenomenon in intelligence analysis [18], information visualization adoption by data analysts [10], and exploratory learning strategies [26]. An advantage of field studies is that the phenomenon being studied is not isolated in a lab but studied in reference to a specific context of human practice. Thus, characteristics of analytic reasoning that are excluded in controlled experiments can be captured by field studies. For example: long-term phenomena, structure of work, division of labor, distributed cognition, peerto-peer interaction, use of diverse information tools, etc. Disadvantages of this method are: studies tend to be long, direct access to domain experts in the workplace is difficult to get, generalizations are difficult to extract and sound theory requires comparative analyses, thus additional research in other field sites, in order to claim generalization.

\section{Pair Analytics}

It does not come as a surprise, given the possibilities and limitations of the reviewed methods, that a thorough understanding of cognitive phenomena requires the combination of two or more methods. The method we propose in this paper builds upon field studies and protocol analysis.

Pair analytics is a method that generates verbal data about thought processes in a naturalistic human-tohuman interaction with visual analytic tools. In pair analytics data about visual analytic reasoning, collaboration in analytical work and analytic discourse can be captured for further analysis. This method is loosely based on "pair programming" from "extreme programming" software development methods [9]. Pair analytics requires a dyad of participants: one Subject Matter Expert (SME) and one Visual Analytics Expert (VAE). The dyad is given one analytical task, one data set, and one computer with one or several visual analytics (VA) tool(s). The VAE has technical expertise in the operation of a suite of VA tools, but may lack the contextual knowledge that would be required to conduct meaningful analysis of the data set $\mathrm{s}$ /he is working on. The SME, on the other hand, has expertise in a specific analytic domain, but VA tools and their features may be unfamiliar to her.

The pairing of SME and VAE is designed to generate a human-to-human dialog that will make explicit mental models and cognitive processes of SME and VAE during their visual analysis. For example, the SME during the analytic interaction may provide expert knowledge to suggest visual comparison of relevant variables, detect patterns and generate or test hypotheses. The interaction of the dyad with the VA tool also generates a human-artifact dialog in which machine-models interact with human mental models. For example, visualizations created by the dyad may result in unexpected outcomes that do not fit into 
existing mental models due to the way the VA tool handles the data. The analytical task and the dataset for pair analytics are selected from previous in-vivo studies of analytical work in the specific domain of expertise of the SME. Selecting a currently relevant analytical task and familiar datasets create a more naturalistic setting for observations of analytical reasoning. Interactions between participants, as well as between VAE and visual analytics tool are captured in video and screen capture.

It is important to note that although participants of the dyad have their own expertise, and the roles of "driver" and "navigator" of the analysis seem to be fixed in VAE and SME, respectively, there is no definite division of labor for cognitive processes. In other words, higher-order cognitive processes, like hypothesis making are not only conducted by the SME, and lower-order cognitive processes, like procedural interaction with the GUI, are not only conducted by the VAE. In fact, switching of roles is a commonly observed practice in pair analytics and it is also a well documented phenomenon in pair programming $[2,4]$.

There are several advantages that Pair analytics offers to cognitive science research in visual analytics with respect to the previously reviewed methods. First, it is a non-intrusive method that takes advantage of the natural and continuous flow of speech necessary to coordinate joint action [6]. According to Herbert $\mathrm{H}$. Clark humans have evolved specific uses of language to coordinate joint activities such as pair analytics. When engaged in joint activities, humans monitor their own and other's mental representations of the activity as well as the current state of the activity in order to coordinate their participation and expectations [6]. Creating and sustaining these shared mental representations, or common ground, is only possible through continuous communication. Since communication between participants flows continuously in pair analytics, a form of joint action, there is no need for a researcher to prompt participants to keep talking, as it is the case in protocol analysis. This is especially important when participants get immersed in the task. The fact that think-aloud protocols are not imposed reduces the possibility of affecting, negatively, reasoning processes, such as discovery and spontaneous insight [3]. Thus, pair analytics provides more complete data about analytical reasoning with less external intervention. This corresponds to our first claim about pair analytics: "it is a more natural way of making explicit and capturing reasoning processes."

Second, collaborative data analysis rather than individual data analysis, as it is common in most of the studies, provides an empirical entry point to study not only individual cognitive processes but also social and distributed cognitive processes of visual analytics. In this aspect, pair analytics gets closer to what could be achieved by field studies. Similar to field studies, pair analytics is conducted in-situ, where domain experts normally conduct their analytical work. Thus, if distributed cognitive processes are commonly conducted in the field site, these will be also made evident in pair analytics data. For example, if peerreview of analytical work and consultations with other domain experts are routine activities of the analysis, then these processes should also be observed or verbalized in pair analytics. Collaborative analytics in real-life settings can also be a middle- to long-term process, especially when analytic tasks are open-ended or exploratory and datasets are not pre-bounded in size and scale [27]. In these cases both, tasks and datasets evolve along with the analysis and a diverse range tools are used to help move forward the analysis. Considering this, pair analytics protocols should adjust to the complexities of analytical tasks and the organizational realities by designing open-ended pair analytic sessions. For example, in another study our laboratory conducted earlier this year, several pair analytic sessions were conducted in-situ with the same dyad over a 2-month period until a report on the analysis was effectively communicated to decisionmakers. In this case the method had to be adjusted to the realities of longer time frames for analytical tasks in the form of a longitudinal study, and the realities of the field, in which collaborative analysis was common. This emphasis on collaborative, in-vivo analytics supports our second claim on pair analytics: "it is an approach to capture social and cognitive processes used to conduct collaborative analysis in real-life settings."

Similar methods to pair analytics, as presented here, have been used before by J.G.Trafton and S.B.Trickett in their studies of cognition in science [36], and in studies of cognition of complex visualizations [33]. Trickett and Trafton method differs from pair analytics in the composition of the dyad and the visual representations used in the studies, which are not "highly interactive," as understood in visual analytics, but correspond to more traditional scientific imagery. However, the method coupled with rigorous coding schemes and cognitive theory has proven useful in advancing models of cognition in scientific work. This has lead us to consider that pair analytics has the same or higher potential for studies of cognition in visual analytics.

\section{Pilot Study}

We designed a pilot study that used pair analytics to generate and collect data, and Joint Action Theory, a 
psycholinguistic theory proposed by Herbert H. Clark, to analyze this data. The research goal of the pilot was to determine the potential of pair analytics to capture to reasoning processes in real-life contexts of visual analysis of massive amounts of data.

The pilot study involved four domain experts in aircraft maintenance engineering and two visual analytics experts from our laboratory. The researchers and the subject matters experts agreed to work on a real analytical task that the aircraft maintenance analysts were working on at the time. The analytical task was open-ended. The objective was generating and testing hypotheses to explain differences of unscheduled aircraft downtime by models of aircrafts in a commercial fleet. The maintenance dataset to be used for the analysis was structured and contained 45 fields and over 90,000 records. One pair analytic session was arranged for each SME, for a total of four sessions. Pair analytic sessions were conducted in-situ, over a period of four weeks in September and October, 2009 , and sessions had an average duration of 2 hours. Tableau, a visual analytics tool, was chosen by the visual analytics experts to be used in the pair analytic sessions. Since the visualizations generated by Tableau during the sessions were going to be line and bar charts, no especial training was required to understand the visual representations of data (Fig.1). However a general introduction about the structure of the data and Tableau was provided to each subject matter expert at the beginning of each session.

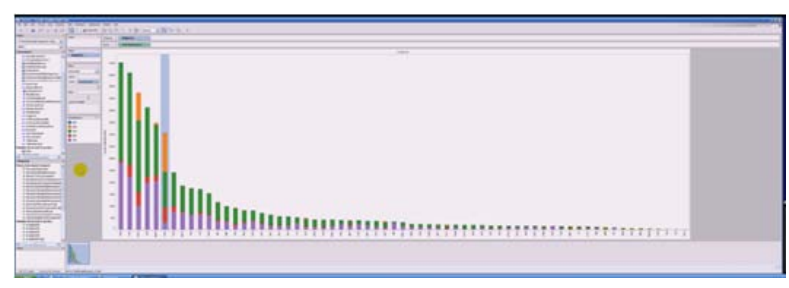

Figure 1. Tableau

Each pair analytics session was video-audio taped, screen captured, and transcribed. The analysis of the data was structured by a coding scheme informed by Joint Action Theory (JAT).

\subsection{Joint Action Theory}

Joint Action Theory (JAT) is a structured, sociocognitive, theory of "language in use" developed by Herbert H. Clark [6]. For Clark, language use is an instantiation of a broader class of human practices: joint actions. In joint actions, individual participatory actions have to be coordinated to produce the intended effect. This implies coordinating content -what the participants intend to do- and processes -the physical and mental systems they recruit in carrying out their intentions-. For example, speaking and listening are individual participatory actions that are coordinated in the joint action of language. To produce a successful joint action, listeners have to attend to a speaker's utterance and identify it, understand it, and decide to take up the speaker's proposal. On the other hand speakers propose joint actions to listeners by uttering speech correctly and presenting appropriate signals with their intended meaning and purpose. Both, speaker's actions and listener's actions are coordinated in terms of content and process.

Joint actions, the fundamental units of analysis in Clark's theory, have several properties and characteristics. They can be coordinated because they divide into phases. A phase is "a stretch of joint action with a unified function and identifiable entry and exit times" [6]. Entry and exit times are what actually participants of the joint action coordinate.

Another characteristic of joint actions is the participant's use of common ground and coordination devices to advance the joint activity. Common ground is shared awareness between participants of a joint action. This shared awareness corresponds to culturally shared knowledge, beliefs and assumptions but also to situated shared knowledge, beliefs and assumptions (situational awareness). Failure in coordinating joint actions commonly occurs due to failures in creating or sustaining this common ground. Coordination devices, which are part of the common ground, give participants of a joint action a rationale for believing they and their partners will converge on the same joint action. Examples of these devices are: explicit agreements (e.g. plans), precedents (e.g. past interactions), conventions, and perceptually salient artifacts (e.g. sheet music). Failure in using the coordination devices also results in failed joint actions.

Since pair analytics is also another form of joint action, we decided to use Clark's joint action theory to analyze the cognitive and social processes used by subjects to structure, coordinate and advance the state of their joint activity. From the theory, we generated a basic coding scheme to capture three cognitive and social phenomena in pair analytics-generated data: coordination of attention, pauses in joint actions due to cognitive workload, and navigation of joint actions between different analytical phases. In the following sections we will present our codes, their operational definitions, and the results obtained from the data.

\subsection{Coordination of Attention}

Based on Clark's theory, we predict that participants of pair analytics will coordinate joint 
attention. Coordinated joint attention is a pre-requisite for successful joint action. Since in joint actions participants continuously propose joint projects to each other, attention of both participants has to be placed in the continuous flow of signals presented by any of them. If attention is not focused on the signal being presented, then the intention behind the signal will not be communicated and the joint action will fail.

This line of reasoning led us to observe the way joint attention is established in pair analytics, the mechanisms participants used to monitor if joint attention is in place and the corrective mechanisms they used to recover joint attention. After preliminary rounds of exploratory analysis on the data, the following codes were proposed and used to capture this phenomenon:

- Directing attention

- Negotiating joint attention

- Confirming joint attention

- Correcting attention

We observed that in pair analytics joint attention was directed to two kinds of signals: signals created by the participants, and signals created by the visual analytics tool. Even though the origin of the signal is different, the presentation of the signal for joint action was usually done by one of the participants. Signals had to be presented by one participant and noticed by the other to advance joint actions. One of the most used mechanisms by participants in pair analytics to direct attention to signals was pointing [5]. In one session, for example, we coded 33 instances of pointing: 15 done by the SME and 18 by the VAE. All of them referred to visual features presented by the visual analytic tool. Participants mostly used two resources for pointing: their body (e.g. finger, hand) and the computer's mouse. Uses of body pointing for coordination of attention have been documented elsewhere [5]. However, uses of mouse pointing as a mechanism to establish joint attention have not been researched systematically. In the pilot study we identified the following uses of the mouse in "directing attention:"

- "directing-to:" the speaker places the mouse on a specific site that points out to a referent being used by him

- "confirming:" the listener places the mouse on a specific site that points out to a referent being used by the speaker

- "moving-around:" the speaker moves the mouse around an area to refer to a conventional place mentioned in her speech

- "traveling-along:" the speaker moves the mouse along a linear segment of the visual to refer to a pattern in a time-series graph

"Directing-to," "moving-around," and "travelingalong" belong to the broader class of "informing location of attention" behaviors. Their use is equivalent to the use of finger pointing in Clark's theory. The mouse pointer is used as a vector that directs the attention of both, speaker and listener, to a physical object in the joint visual space. Attention to this signal and proper identification of the object being signaled is necessary to eliminate ambiguities in the use of demonstrative pronouns (e.g. this, that) and adverbs (e.g. here, there) when referring in the speech to visible objects in the GUI. In all of these behaviors, the mouse is appropriated as a communicational artifact between the two participants rather than as an interactional artifact to trigger events in the visual analytics tool. The difference between these three behaviors lies on the object being indicated. In the first case, it is a discrete object (e.g. a peak in the line chart, a label, a visible state of a variable in the filtering of data). In the second case, it is an area (e.g. a whole chart in a series of several visible charts, objects that are not visually present at the time but that there were in that area or that will be in that area). In the third case, it is a pattern of the data (e.g. declining values of a variable). The following is an excerpt taken from the transcript of one of the sessions that illustrates a "directing-to" kind of behavior (in bold):

\author{
SME: okay \\ VAE: [clicks on the orange section of the bar "HOU"] \\ lots leaving from Houston \\ SME: So, that's interesting \\ VAE: yeah ... [clicks on the orange section of "DAL"] \\ lots leaving from Dallas [clicks on label of the bar \\ "DAL" on the $\mathrm{X}$-axis] \\ SME: yeah
}

The mouse-pointing behavior "confirming," which belongs to the broader class of behaviors "confirming joint attention," is the most interesting one since it is the listener who executes it. In the case of pair analytics, since the mouse was controlled all the time by the VAE, this behavior was always produced by the VAE acting as the listener. While the speaker (SME) was referring to an object on the visual representation, sometimes pointing at it, sometimes not, the listener (VAE) in control of the mouse would use the mouse to point at the object being referred to. This behavior produces a visual cue that informs that the listener's attention is placed where the speaker's attention is located. Once the visual cue is evident corrective mechanisms on attention can be applied if joint attention is not placed on the same referent. The following is another excerpt that illustrates a successful instance of confirming joint attention:

SME: so ... looking at that ... let's see the ... 200s are the orange 
VAE: [moves the mouse over one of the bars with a visible orange stack] [inclines his head to read the vertical labels] yeah, so ...

These results from the pilot study demonstrate that data about social and cognitive processes used to manage joint attention can be captured by pair analytics supporting our second claim about this method. These results are preliminary but a model of how joint attention operates in collaborative analytics and its uses is now in the making.

Generating knowledge and models about individual and social cognition in collaborative analytics is necessary to provide sound scientific principles that can be used to guide design and evaluation of visual analytics tools. Some initial work in this area using ideas about uses of pointing in joint actions has already influenced the design of features in asynchronous, collaborative visual analytic tools. For example Heer and Agrawala [14] designed a system that allows analysts to place marks on views of data and link these marks to textual analytical comments on collaborative spaces like fora. This feature uses these marks to direct attention from textual annotations to specific aspects of the view ("directing to" behavior). The textual remarks, necessary to describe the marked referent to collaborators, are now replaced with a link to the marked referent reducing the number of words used to communicate analysis. However, the richness of joint action theory resides on the natural evolution of human language in synchronous face-to-face interaction. Thus, synchronous scenarios of collaboration can benefit even more from using methods for indicating such as finger or mouse pointing. For example, a collaborative visual analytic system that allows analysts to work simultaneously on the same tool could include a speech channel and differentiated pointers assigned to each of participants involved (e.g. hands, colored-arrows, or a different metaphor). In real-time interaction, participants would see their own and others' pointers when they are being used to indicate a reference in their speech. Expected advantages of these systems, according to JAT, would be less ambiguity, less errors in communication, and more analysis being done in an amount of time.

\subsection{Pauses in joint actions and cognitive workload}

Applying JAT to the analysis of pair analytics data requires organizing the human-to-human dialogue in phases. Clark's theory predicts that participants will coordinate entry and exit times of phases by using verbal or non-verbal markers (see next section). While working on the structuring of the pair analytic sessions in phases, we noted that some joint activities were temporarily paused by one of the participants in order to engage in time or cognitive demanding tasks. These individual, participatory activities demanded immersion of one of the participants in the execution of a specific task. In all of the instances observed, the task involved direct or indirect interaction with the visual analytic tool. Once the task was finished, the participant would resume the paused joint activity. For example, in some occasions the VAE would get a request from the SME to create a non-trivial view of the data. The VAE would then interact with the application in solo mode and conduct several steps to produce the intended view. Once done, the VAE would resume interactions with the SME. The SME, on the other hand, also engaged in similar kinds of behavior. When observing a new view of the data, the SME would stop interacting with the VAE to observe the features of the view, and return to join action afterwards. The following excerpt from one of the sessions illustrates the VAE in one of these pauses:

VAE: [clicks on "new worksheet," clears the view area, and generates another thumbnail]

new worksheet ... not new,

[rightclicks on the last thumbnail, selects "Delete

Sheet," restores the last view]

duplicate

[clicks on "Duplicate sheet," generates a thumbnail

keeping the previous view on screen]

... and we want to look at "available minutes"

[moves the mouse to the Measure frame, moves the vertical scrollbar until he locates "Available minutes"]

It is important to note that these pauses are not interruptions since both participants are still on-task and advancing the joint activity. These pauses can be better characterized as delays caused by the cognitive demands on participants generated by the ongoing task. The more cognitive demanding the task is, the longer the pause will be. The cognitive resources seem to switch from the human-to-human interaction to the human-to-computer interaction. Therefore, these pauses in joint actions or between joint actions can be potential candidates to capture empirically instances of high cognitive workload produced by visual analytic tools [30].

Another related finding was that different than protocol analysis, in which participants stop thinkingaloud when immersed in a task, in pair analytics participants do not stop talking, even when immersed in highly-demanding cognitive tasks, such as the pauses in joint action. On the contrary, participants go along with the task, thinking-aloud or "self-talking" all the time. A possible explanation for this behavior is 
that self-talk in pair analytics may create a temporary isolation from dialogue to concentrate in the humancomputer interaction while informing the other participant that a delay is in place and how the task is progressing. Self-talk helps prevent external noise or attempted joint actions produced by the other participant from interrupting the execution of the individual task. In any case, self-talk provides valuable data about cognitive processes being performed by participants immersed in cognitive demanding tasks. This data is generated in a natural, non-invasive way supporting our first claim that pair analytics is a more natural way of making explicit and capturing reasoning processes.

\subsection{Navigation between Analytic Phases}

JAT establishes that the use of project markers to distinguish among phases of activity (e.g. "mhmm," "okay," "right," and "oh") is determined by what the speakers are trying to accomplish, rather than simply marking turns in the dialog. Bangerter \& Clark [1] identify three main phases of activity in a wellstructured task, A) the overall session, B) the identification of components, and C) the asking and answering of questions. Their study demonstrates that continuers such as "mhmm" and "yeah" mark horizontal transitions in a joint activity, whereas interjections such as "okay" and "all right" mark vertical transitions. We also used the pilot to study project markers in the context of a pair analytics session to see if the combination of visual analytic software and a pair analytic task change the way project markers are used and/or understood in the negotiation of joint activities.

In contrast to Bangerter \& Clark, the pair analytics study was conducted on tasks that were not scripted or well-defined, but rather, reflected the messy reality of industrial data analysis problems. Nevertheless, the activities inside the sessions fall into three general categories that map onto those outlined by Bangerter $\&$ Clark. The sessions contain an overview of the pair analytics goals (A), an examination of the components in the data (B), and the posing of analytical questions or problems (C). While (A) is fairly contained within the first 5-8 minutes of the sessions, (B) and (C) can and do overlap as components of the data and visualization tool are continually being identified and re-defined while the questions and problems are posed.

Early in the analysis, it became clear that the explanatory dialog of (B) and the analytical dialog of (C) have distinct differences. Turn-taking is very apparent, even rhythmic, in explanatory dialog, whereas simultaneous and overlapping speech and long silences often characterize analytical dialog. In addition, markers such as "right" and "okay" can be accompanied by intonation and bodily signals that change their function from vertical - moving from one subtask to another, to horizontal - serving as a continuer for the current speech act. For example, "right", when accompanied by small, repetitive head nods and an upward intonation functions as an acknowledgment token and continuer (horizontal marker). When "right" is uttered with no head nod, or a large head nod possibly followed by a few small nods, and a downward intonation, it serves as an agreement token and vertical marker.

In addition, body motion can serve as a project marker without the accompanying speech. In our data, large body motions such as moving the head and torso away from the display, turning to gaze directly at the speaker, or turning away from the speaker form similar functions as verbal project markers. Moving back and away from the screen can indicate the shift of attention to a new problem or sub-problem. Gazing at the speaker directly (when they are generally seated sideby-side) can serve as a continuer. Turning away from the speaker draws attention away from their speech and shifts attention to a new problem or sub-problem. This points to more questions about how studies measuring analytical joint activities should be designed. It is clear from these preliminary findings that measures of body motion and intonation should accompany the study of verbal project markers in transitions between analytical tasks. From a method perspective, these findings demonstrate again how pair analytics supports our claim that it captures social and cognitive processes, such as the use of linguistic markers, used to conduct collaborative analysis in real-life settings.

\section{Limitations of Pair Analytics}

As with any other method used to capture analytic reasoning in visual analytics, pair analytics has its limitations. Similar to field studies, pair analytics requires direct access to domain experts in the workplace, which is not easy to get. This access is especially necessary to identify SMEs, relevant tasks and datasets for the sessions. Since data is collected only during analytical sessions using the visual analytics tool, uses of other resources to support the analysis, other actors involved in the analysis and other moments of analysis that are not present in the sessions cannot be captured by this method.

Another consequence of pair analytics being an invivo study is that the variables that influence the phenomenon of study are not controlled. Therefore, it is not possible to make bold generalizations but rather tentative hypothesis about the phenomenon. These hypotheses have to be tested by other methods, such as 
controlled experiments or comparative studies, in order to advance towards a more encompassing scientific theory.

Pair analytics, just as protocol analysis, is labor intensive and requires transcription of the data because it relies on verbal reports from the participants. Depending on the theory being used to guide the analysis, such as JAT in our case, it may even require additional work in transcribing interactions with the interface or in using logging mechanisms to provide data about the interaction with the visual analytic tool.

\section{Conclusions}

Our pilot study in this paper provides an example of using pair analytics to capture reasoning processes in a collaborative scenario. We found this method valuable in terms of providing data to studying cognition in visual analytics whether the focus was on individual cognitive processes or in social cognitive processes. Based on our experience using pair analytics we claimed that this method provides: (1) a more natural way of making explicit and capturing reasoning processes and (2) an approach to capture social and cognitive processes used to conduct collaborative analysis in real-life settings. To support these claims we used pair analytics-generated data in a pilot study to capture three phenomena: coordination of joint attention, pauses in joint actions as indicators of cognitive workload, and navigation between analytical phases. Our pilot results indicate that pair analytics provides data that captures cognitive phenomena advancing claims of validity for this method. Currently, we are limiting the analysis of pair analytics data to the scope of joint action theory, which focuses on psycho-linguistic processes that structure, coordinate and advance pair analytics. However, other cognitive science theories can be used to code and analyze pair analytics data. Further work is also necessary to demonstrate the validity and reliability of pair analytics, and its advantages with respect to methods like protocol analysis. This will require the design of experiments.

\section{Acknowledgments}

This research was supported by NSERC STPGP 336506 "Visual Analytics for Safety and Security," MITACS ACCELLERATE BC "Program to Evaluate and Improve Visual Analytic Processes for Analyzing Maintainability, Reliability and Safety Data," and "Boeing Support for Visual Analytics in Canada" (Fisher subgrant).

\section{References}

[1]Bangerter, A. and H.H. Clark, "Navigating joint projects with dialogue," Cognitive Science, vol. 27, no 2, 2003, pp. 195-225.

[2]Bryant, S., P. Romero and B. Boulay, "Pair programming and the mysterious role of the navigator," International Journal of Human-Computer Studies, vol. 66, no. 7, July 2008, pp. 519-529.

[3]Chang, R., C. Ziemkiewicz, T. M. Green, and W. Ribarsky, "Defining insight for visual analytics," IEEE Computer Graphics and Applications, vol. 29, no. 2, 2009, pp. 14-17.

[4]Chong, J. and T. Hurlbutt, "The Social Dynamics of Pair Programming," IEEE 29th International Conference on Software Engineering (ICSE'07), Minneapolis, MN, May 2026, 2007.

[5]Clark, H.H., "Pointing and Placing," In: S.Kita (ed.), Pointing: Where language, culture and cognition meet, Erlbaum, Hillsdale, NJ, 2003, pp.243-268.

[6]Clark, H.H., Using Language, Cambridge University Press, Cambridge, MA, 1996.

[7]Dunbar, K., "How scientists think: online creativity and conceptual change in science." In: T.B. Ward, S.M. Smith and S. Vaid (eds). Conceptual Structures and Processes: Emergence, Discovery and Change. Washington, DC: APA Press. 1996.

[8]Ericsson, K.A. and H.A. Simon, Protocol analysis: Verbal reports as data, MIT Press, Cambridge, MA, 1993.

[9]Gallis, H., E. Arisholm and T. Dyba, "An initial framework for research on pair programming," In: Proceedings of the International Symposium on Empirical Software Engineering, 2003.

[10]Gonzales, V. and A. Kobsa, "A Workplace Study of the Adoption of Information Visualization Systems," Proc. IKNOW: Third International Conf. Knowledge Management, 2003, pp. 92-102.

[11]Gorman, M.E., "Introduction to Cognition in Science and Technology," Topics in Cognitive Science, vol. 1, no. 4, Oct. 2009, pp. 675-685.

[12]Green, T.M., D.H. Jeong and B. Fisher, "Using personality factors to predict interface learning performance," In: Proceedings of Hawaii International Conference on System Sciences 43, Jan. 5-8, Koloa, Hawaii, 2010 . 
[13]Green, T.M., W. Ribarsky, and B. Fisher, "Building and applying a human cognition model for visual analytics," Information Visualization, vol. 8, no. 1, 2009, pp. 1-13.

[14]Heer, J. and M. Agrawala, "Design considerations for collaborative visual analytics," Information Visualization, vol. 7 , no. 1,2008 , pp. 49-62.

[15]Hutchins, E., Cognition in the Wild. Cambridge, MA: MIT Press, 1995.

[16]Irani P., and C. Ware, "Diagramming Information Structures Using 3D Perceptual Primitives," ACM Trans. Computer-Human Interaction, vol. 10, no. 1, pp. 1-19, 2003.

[17]Jeong, D.H., W. Dou, F. Stukes, W. Ribarsky, H.R. Lipford and R. Chan, "Evaluating the Relationship Between User Interaction and Financial Visual Analysis," IEEE Symposium on Visual Analytics Science and Technology, Atlantic City, Columbus,OH, October 19-24, 2008, pp. 8390 .

[18]Johnston, R., Analytic Culture in the US Intelligence Community: An Ethnographic Study, Center for the Study of Intelligence, Central Intelligence Agency, 2005.

[19]Kang, Y., C. Gorg and J. Stasko, "Evaluating Visual Analytics Systems for Investigative Analysis: Deriving Design Principles from a Case Study," IEEE Symposium on Visual Analytics Science and Technology, Atlantic City, NJ, Oct. 12-13, 2009, pp. 139-146.

[20]Latour, B. and S. Woolgar, Laboratory Life: The Construction of Scientific Facts, Sage Publications, Beverly Hills, 1979.

[21]Militello, L.G. and R.J.B. Hutton, "Applied cognitive task analysis (ACTA): a practitioner's toolkit for understanding cognitive task demands," Ergonomics, vol. 41, no. 11, 1998, pp.1618-1641.

[22]Nardi, B., Context and Consciousness: Activity Theory and Human-computer Interaction. MIT Press, Cambridge, MA. 1996

[23]Pirolli, P. and S.K. Card, "Information Foraging," Psychological Review, vol. 106, no. 4, 1999, pp. 643-675.

[24]Pirolli, P. and S.K. Card, "The Sensemaking Process and Leverage Points for Analyst Technology as Identified Through Cognitive Task Analysis," In: Proceedings of International Conference on Intelligence Analysis, McLean, VA: Mitre, May 2005.

[25]Ribarsky, W., B. Fisher and W.M. Pottenger, "Science of Analytical Reasoning," Information Visualization, vol. 8, no. 4, 2009, pp. 254-262.
[26]Rieman, J., "A Field Study of Exploratory Learning Strategies," ACM Trans. Computer-Human Interaction, vol. 3, pp. 189-218, 1996.

[27]Robertson, G., D. Ebert, S. Eick, D, Keim and K. Joy, "Scale and Complexity in Visual Analytics," Information Visualization, vol. 8, no. 4, 2009, pp. 247-253

[28]Saraiya, P., C. North and K. Duca, "An insight-based methodology for evaluating bioinformatics visualizations," IEEE Transactions on Visualizations and Computer Graphics, vol. 11, no. 4, Jul-Aug 2005, pp. 443-456.

[29]Saraiya, P., C. North, V. Lam, and K. Duca, "An insightbased longitudinal study of visual analytics," IEEE Transactions on Visualizations and Computer Graphics, vol. 12, no. 6, Nov-Dec 2006, pp. 1511-1522.

[30]Scholtz, J., "Beyond Usability: Evaluation Aspects of Visual Analytic Environments," IEEE Symposium on Visual Analytics Science and Technology, Baltimore, MD, Oct. 31Nov. 2, 2006, pp. 145-150.

[31]Schooler, J.W., S. Ohlsson, and K. Brooks, "Thoughts beyond words: When language overshadows insight," Journal of Experimental Psychology: General, vol. 122, no. 2, June 1993, pp. 166-183.

[32]Suchman, L., Plans and Situated Action: The Problem of Human-Machine Communication. Cambridge University Press, New York, 1987.

[33]Trafton, J.G., S.S. Kirschenbaum, T.L. Tsui, R.T. Miyamoto, J.A. Ballas, and P.D. Raymond, "Turning Pictures into Numbers: Extracting and Generating Information from Complex Visualizations," International Journal of Human Computer Studies, vol. 53, no. 5, 2000, pp. 827-850.

[34]Trickett, S.B. and J.G. Trafton, “,What if ...': The Use of Conceptual Simulations in Scientific Reasoning," Cognitive Science, vol. 31, no. 5, Sep-Oct 2005, pp. 843-875

[35]Trickett, S.B., J.G. Trafton, L.D. Saner and C.D. Schunn, "II don't know what's going on there": The Use of Spatial Transformations to Deal With and Resolve Uncertainty in Complex Visualizations," In: M.C. Lovett and P. Shah (eds.), Thinking with Data, New York, NY: Lawrence Erlbaum Associates, 2007, pp. 65-86

[36]Trickett, S.B., W.T. Fu, C.D. Schunn and J.G. Trafton, "From dipsy-doodles to streaming motions: Changes in representation in the analysis of visual scientific data." In: Proceedings of the 22nd Annual Conference of the Cognitive Science Society. Mahwah, NJ: Erlbaum, 2000.

[37]Wright, W., D. Schroh, P. Proulx, A. Skaburskis, and B. Cort. "The sandbox for analysis: Concepts and methods," In ACM CHI, April 2006, pp. 801-810. 